\title{
Ultrasound on Seedling Growth of Wheat under Drought Stress Effects
}

\author{
Haiyan Ran, Liyan Yang, Yanling Cao* \\ Department of Life Science, Shanxi Normal University, Linfen, China \\ Email:
}

Received 15 June 2015; accepted 11 July 2015; published 14 July 2015

Copyright (C) 2015 by authors and Scientific Research Publishing Inc.

This work is licensed under the Creative Commons Attribution International License (CC BY).

http://creativecommons.org/licenses/by/4.0/

\section{(c) (i) Open Access}

\begin{abstract}
This study used different intensity ultrasound treatments, wheat seedlings in the germination energy, germination rate, growth potential, root length, and number of lateral roots optimal radiation dose selection. Studied under drought stress were the optimal dose of radiation treatment of wheat seedling leaf relative water content (RWC), protein content, Methane Dicarboxylic Aldehyde (MDA) content, relative conductivity, Superoxide dismutase (SOD) activity, and the impact of amylase activity. Studies have shown that after ultrasonic treatment enhanced drought resistance of wheat seedlings, alleviate drought stress on wheat seedling injury to $65 \mathrm{~W} 15$ min ultrasonic treatment works best.
\end{abstract}

Keywords

Drought Stress, Ultrasound, Drought Resistance

\section{Introduction}

Wheat, regarded as one of the entire world's most important food crops, is widely grown in the world. About a third of the entire world's population mainly feeds on wheat, of which yield directly relates to the world food security. However, seventy percent of the entire world's wheat growing region distributes in arid and semiarid region [1] [2]; drought has become one of the important factors that threaten wheat production. With the aggravation of the global water crisis, the seriousness of the problem is further aggravated [3]. And if the wheat can resist drought stress without affecting its production, the solution of the problem on food for the whole world will be a breakthrough.

In recent years, the application of ultrasonic technology in the correlative industry is more and more widely, which is also very widely used in agriculture [4] [5]. Some studies have shown that low intensity ultrasound has effects on promoting grain crop seed germination and increasing yield [4].

${ }^{*}$ Corresponding author. 
This research treats wheat seed with the ultrasonic and measure morphology and related physiological indexes of wheat [6], such as relative water content, relative electric conductivity, protein content, SOD activity and MDA content. Beyond that, it also studies the ultrasonic effects on the drought resistance of wheat under drought stress. In the experiment, we will select the proper coefficient of ultrasonic to provide a new and feasible method for alleviating the effects of drought on wheat seedling.

\section{Materials and Methods}

Wheat seeds (Triticum aestivum L. cv. Lin optimal 2069) were selected as the materials.

\subsection{Filtration of Ultrasound Doses}

First, select plump and consistent to the size of the wheat seed. According to the existing research, we process the wheat seed with $195 \mathrm{w}, 130 \mathrm{w}, 65 \mathrm{w}$ ultrasonic treating $30 \mathrm{~min}$ and $15 \mathrm{~min}$ respectively. The distance between the ultrasound Emitters and the seed was $10 \mathrm{~cm}$. Meanwhile let unprocessed wheat seeds and the processed wheat grow together. Finally, measure wheat germination potential, germination rate, growth potential and root length after 3 day cultivation.

\subsection{Drought Treatment}

Give three days alike treatments that drought stress, soil conditions, temperature, fertilizer and so on, to the optimal treatment group and control group of wheat seedlings in growth after a week.

\subsection{Measurement of Growth Index}

Measure wheat germination potential, germination rate, growth potential, root length, lateral root number after 3 day cultivation. Germination rate indicate the seed germination capacity, using the type calculation to measure: germination rate $=$ (germinating seed grain number divide selected seed grain number) by $100 \%$. Germination potential indicates the germination of seeds germination speed and germination uniformity, the strength of the seed vigor, using the type calculation: the germination = (germinating seed grain number divide the selected seed grain number in certain time) by $100 \%$. Growth potential is pointed to plant growth and development degree, while the leaf area is an important symbol of crop growth potential strength, using the type calculation to measure:square meters leaf area multiply plant height with a development period. so we respectively use direct method to calculate and measure various index.

\subsection{Measurement of Physiological Indexes}

Measure the physiological indexes of wheat seedlings, which is processed by drought. Measurement of the relative water content (RWC) and relative electric conductivity respectively refer to Zhi and Farshadfar's method [7] [8], Ai Kuichen method [9]. The measurement of MDA content use thiobarbituric acid (TBARS) method [10] [11], using $\mu \mathrm{mol} \cdot \mathrm{g}^{-1} \mathrm{FW}$ to indicate MDA content. The measurement of protein content refer to Wei [12], expressing protein content in $\mathrm{mg} / \mathrm{mL} \mathrm{FW}$; The measurement of SOD activity refer to Zhu [13], regarding that restrain nitroblue tetrazolium (NBT) light to restore by $50 \%$ per minute as one unit of enzyme activity (U), and expressing the activity of enzyme in $\mathrm{U} \cdot \mathrm{g}^{-1} \mathrm{FW} \cdot \mathrm{min}^{-1}$. The measurement of amylase refer to Zhu [13], expressing the activity of amylase in $\mathrm{mg} / \mathrm{g} \cdot \mathrm{min}$.

\section{Results}

\subsection{The Influence of Different Ultrasonic Processing on Wheat Seedling Growth}

As shown in Figure 1 and Figure 2, compared with the control group, wheat seedling germination potential, germination rate, growth potential and root length were significantly reduced after the $195 \mathrm{w}$ ultrasonic processing; By the $130 \mathrm{w}$ ultrasonieat processing, wheat seedling germination potential, germination rate, growth potential and root length were slightly lower than the control group. By the $65 \mathrm{w}$ ultrasonic processing after 15 min, wheat seedling germination potential, germination rate, growth potential and root length were higher than that of control group, which showed that low intensity ultrasound processing can promote the germination of wheat seed. 


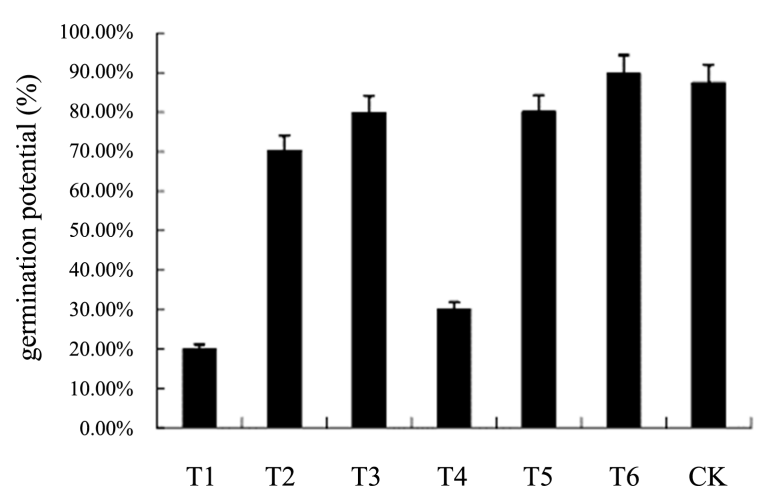

(a)

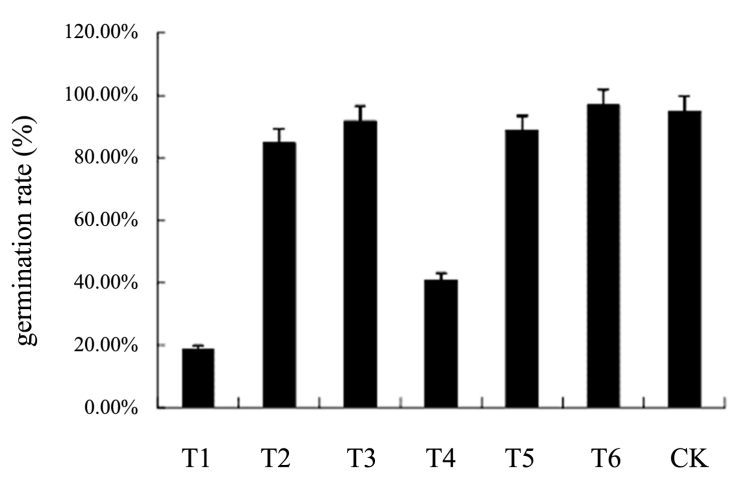

(b)

Figure 1. The influence of germination potential (a) and germination rate (b) by different treatment.

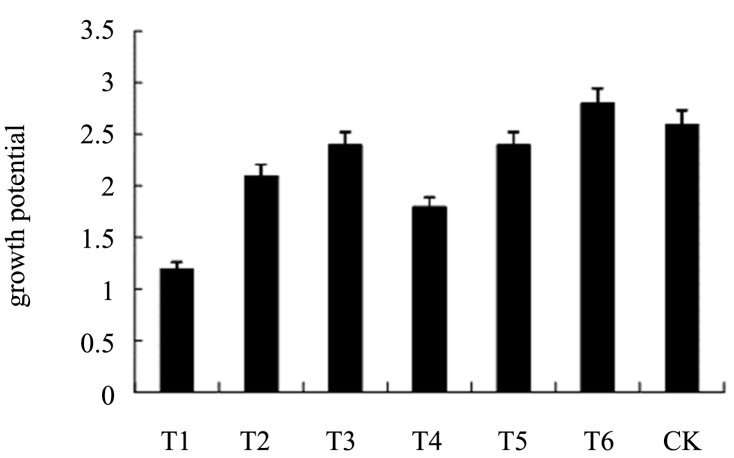

(a)

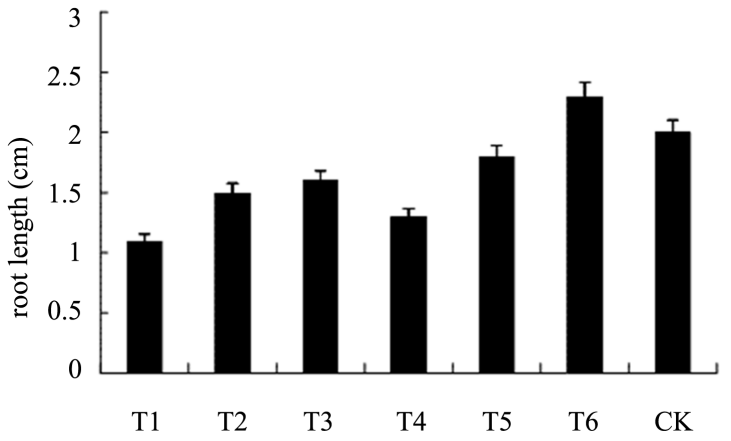

(b)

Figure 2. The influence of growth potential (a) and root length (b) by different treatment.

According to the above results, we could find: compared with other groups, $65 \mathrm{w}, 15$ min processing wheat seedling had highly ability in growth, but not necessarily the optimal dose and time, which still can was selected as the experimental conditions. Then, the control group and $65 \mathrm{w}, 15 \mathrm{~min}$ processing wheat seedling were in drought stress at the same time, and measured corresponding physiological indexes to study the dose of ultrasonic effect on wheat seedling under drought stress .

\subsection{The Influence of $65 \mathrm{w}, 15 \mathrm{~min}$ Ultrasonic Processing on the Relative Water Content and Protein Content of Wheat Seedlings under Drought Stress}

A large number of studies have shown that high leaf relative water content of wheat has higher drought resistance capability [7]-[14]. As shown in Figure 3(a), apparently, the experimental group of wheat leaf relative water content is higher than the control group, the increasing quantity is approximately thirty percent. Drought stress could restrain protein synthesis, induce protein degradation, and reduce total protein content in plants, which had close relationship with the senescence of plant .Meanwhile it was also a physiological performance of plant damage under drought stress [15]. As shown in Figure 3(b), compared with the control group, 65 w ultrasonic processing of wheat seedlings increased protein content after $15 \mathrm{~min}$, which could relieve plant senescence.

\subsection{The Influence of $65 \mathrm{w}, \mathbf{1 5}$ min of Ultrasound Processing on the Relative Conductivity and MDA Content of Wheat Seedling under Drought Stress}

Conductivity reflect the stand or fall of plant membrane system. When the plant was in stress or damage, membrane protein damage, membrane easily broke, even cytosol extravasation, which led to the increase of relative electrical conductivity [9]. So the relative electrical conductivity could be used as a physiological index of 


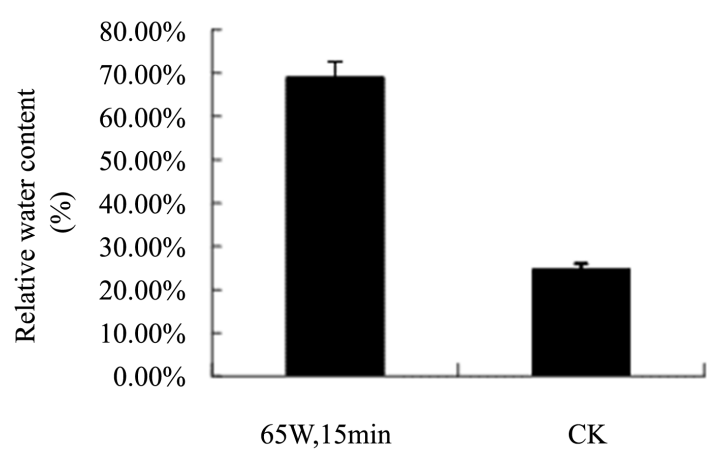

(a)

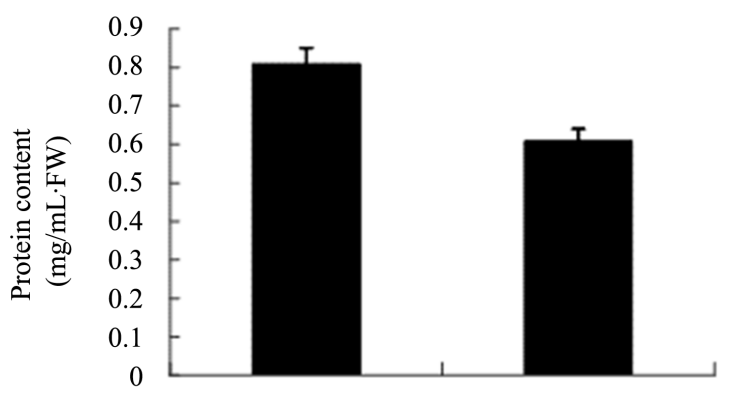

$65 \mathrm{~W}, 15 \mathrm{~min}$

CK

Figure 3. The Relative water content and Protein content of wheat seedling leaf.

membrane damaged. As shown in Figure 4(a), the relative conductivity of the experimental group were lower than those of control group, which indicate membrane system was in good condition, but the effect was not very significant. Malondialdehyde (MDA) is one of membrane lipid peroxide . The generation of peroxide makes the membrane proteins in a state of permanent association, thus it can limit the movement of membrane protein. Increasing the MDA content is an important sign of membrane structure damage [16]. As shown in Figure 4(a), MDA content in the experimental group was lower than control group, which showed that low doses of ultrasound could improve the wheat seedling cell membrane fluidity to a certain extent.

\subsection{The Influence of 65 w, 15 min of Ultrasound Processing on SOD Activity and Amylase Activity of Wheat Seedling under Drought stress}

Superoxide dismutase (SOD) containing metal ions is a kind of eliminable enzymes of active oxygen, which catalyze super oxygen anion disproportionation reaction, and generate $\mathrm{H}_{2} \mathrm{O}_{2}$ and $\mathrm{O}_{2}^{-}$. Thereby, SOD can eliminate the damage of $\mathrm{O}_{2}^{-}$to the cells [17]. As shown in Figure 5(a), compared with the control group, wheat seedling of $65 \mathrm{w}$ ultrasonic processing could increase SOD activity after $15 \mathrm{~min}$, and effectively relieve the damage of super oxygen anion free radical that is produced by the drought stress to cells. Seeds store a lot of starch that can provide nutrients for the growth of embryo through the action of amylase catalyzing starch hydrolysis, when seeds germinate [18]. The amylase activity is a close relationship between seed germination and seedling growth. As shown in Figure 5(b), amylase activity about $480 \mathrm{mg} / \mathrm{g} \cdot \mathrm{min}$ of wheat seed in the experimental group was higher than the control group, which showed that $65 \mathrm{w}$ ultrasonic treatment is beneficial to seed germination and seedling growth of wheat after $15 \mathrm{~min}$.

\section{Discussion}

Ultrasound as a form of stress role has important effects on plant growth and development [5]. Ultrasonic treatment can influence the growth of some organs of plant. Research found that a little of ultrasound could stimulate cell division, medium dose of ultrasound could restrain cell division, a large number of ultrasonic could cause cell death when ultrasonic process seeds. Study also found that spinach and cabbage seeds germination rate was increased with ultrasonic processing. Mild ultrasonic can make the plant root cell to vigorously divide, enhance plant growth ability, promote the plant to strike roots; which could decrease the respiration intensity of annual plants, while increase the respiration intensity of plants for two years.

Study found that a large number of ultrasound could restrain the growth of the plant, a small amount of ultrasound could improve the germination, growth of plants, root elongation, when treat wheat seeds with different doses of the ultrasonic. Study also found dealing plants with $65 \mathrm{w}$ ultrasonic $15 \mathrm{~min}$ could work best.

Since water weight of saturation organization is stable, the change of the relative water content is more sensitive than the water content of fresh weight when the plant lacks water. Relative water content of plant can represent a kind of ability of holding water and avoiding dehydration, which can regard as a kind of index of plant drought resistance [7]. Protein content can reflect the degree of aging cell. If protein content is low, the cell's aging degree is serious. This study showed that wheat seedling leaf with $65 \mathrm{w}$ ultrasonic treatment for 15 


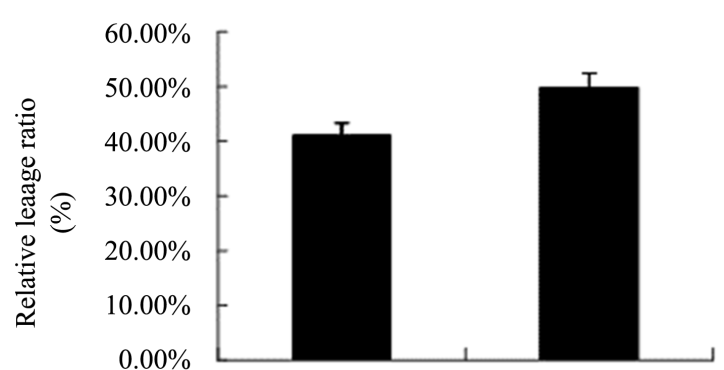

$65 \mathrm{~W}, 15 \mathrm{~min}$
CK

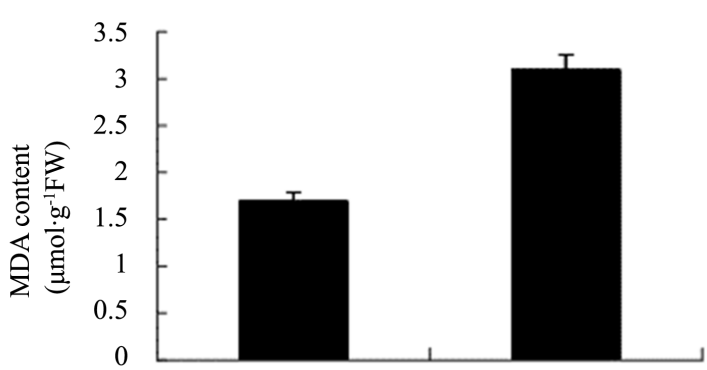

$65 \mathrm{~W}, 15 \mathrm{~min}$
CK

(a)

(b)

Figure 4. The relative conductivity Figure ure and MDA content of wheat seedling leaf.

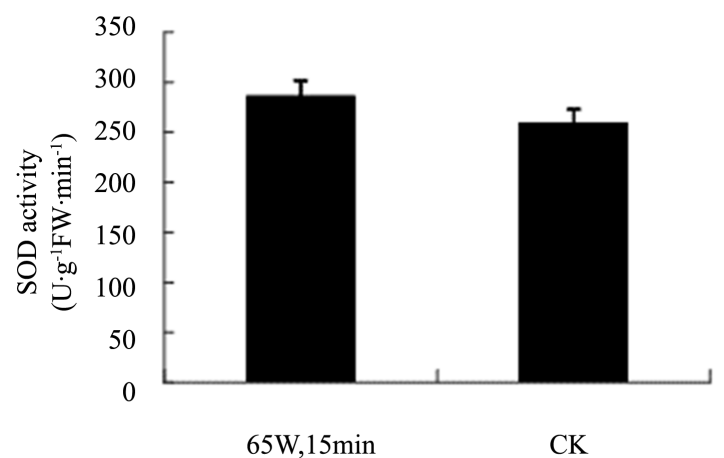

(a)

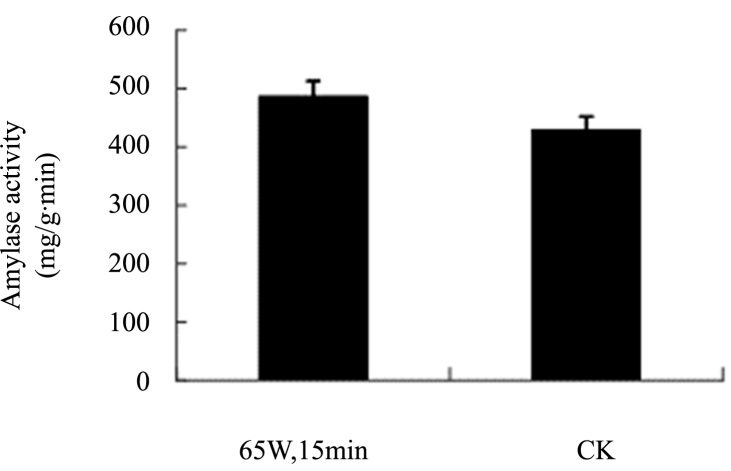

(b)

Figure 5. The SOD activity and amylase activity of wheat seedling leaf.

min relative water content and protein content were significantly higher than control group, which could demonstrate that low doses of and protein content, improve the ability of plant resistance to drought stress.

Plant in adversity, unsaturated fatty acid in the cell plasmalemma produce MDA through peroxidation, which may damage to the plasmalemma system and even may make the quantity of electrolyte inside the cytoplasm leakage increase. So MDA content can reflect one of the sign of damaging to membrane structure [16]. The permeability of plasmalemma can express the degree of membrane injury and degeneration. When the permeability of plasmalemma is big, the degree of membrane denatured is high [9]. This study showed that wheat seedling leaf of $65 \mathrm{w}$ ultrasonic treatment for $15 \mathrm{~min}$ MDA content and relative conductivity significantly reduced than the control group, which Indicated that low doses of ultrasound could reduce blade MDA content and relative conductivity in different degrees to protect the plasmalemma structure from destruction. Thus Low doses of ultrasound could prove the ability of plant resistance to drought stress.

Protected enzyme that can elimate active oxygen system in plants is one of the important enzymes. The activity of SOD increasing is one of the behavior to cope with adversity stress. Amylase activity can reflect seed germination vigor. Wheat seedling with $65 \mathrm{w}$ ultrasonic treatment for $15 \mathrm{~min}$ leaf SOD activity and amylase activity increased than the control group, which showed that low doses of ultrasound could increase the protective enzyme activity and amylase activity of wheat seedlings to effectively slow the damage of drought stress on wheat seedlings.

\section{Conclusion}

In this work, the wheat seedlings were treated by the ultrasonic. And then we measured the morphology and related physiological indexes of wheat seedlings, such as relative water content, relative electric conductivity, protein content, SOD activity and MDA content. And this research also studied the effects of ultrasonic on the drought resistance of wheat under drought stress. Our results showed that after ultrasonic treatment enhanced drought resistance of wheat seedlings, alleviate drought stress on wheat seedling injury to $65 \mathrm{w} 15$ min ultrason- 
ic treatment works best.

\section{References}

[1] Zhang, L.G. and Deng, X.P. (2000) Advances in Studies on Physiology and Biochemistry of Wheat Drought Resistance. Agricultural Research in the Arid Areas, 18, 87-92.

[2] Yang, Z.G., Zhang, C.J., Ji, T.H., Guo, J.W., Meng, L.M. and Zhang, K. (2007) Study on Resistance Drought Identify Method and Evaluation Index of Wheat IV, The Comparative Study on Resistance Drought Index of Wheat in Germ ination. Chinese Agricultural Science Bulletin, 23,173-176.

[3] Wu, J.S. (1994) The Problem of the Potential of Water and Soil Resources in the World and the Population of Grain. World Agriculture, 2, 256-260.

[4] Yang, H.B., Ding, W.M., Chen, K.J. and Ding, Y.Q. (2004) Application of Ultrasonic Technique in Agriculture-Actuality and Prospect. Journal of Agricultural Mechanization Research, 1, 365-370.

[5] Zheng, W.X., Wu, S.J. and Yang, Y. (2008) Ultrasound Application in Agriculture and Prospect. Agricultural Outlook, 1, 115-119.

[6] Bai, Z.Y., Li, C.D., Sun, H.C. and Zhao, J.F. (2008) Principal Component Analysis and Comprehensive Evaluation on Physiological Indices of Drought Resistance in Wheat Substitution. Scientia Agricultura Sinica, 41, 4264-4272.

[7] Bai, Z.Y., Li, C.D. and Sun, H.C. (2008) Effect of Drought Stress on Relative Water Content and RWL of Flag Leaves in Wheat Chromosome Substitution Lines. Acta Agriculturae Boreali Sinica, 23, 62-65.

[8] Farshadfar, E., Koszegi, B. and Tischner, T. (1995) Substitution Analysis of Drought Tolerance in Wheat (Tritwum aestivum L.).Plant Breeding, 114, 542-544. http://dx.doi.org/10.1111/j.1439-0523.1995.tb00853.x

[9] Chen, A.K., Han, R.H., Li, D.Y., Lin, L.L., Luo, H.X. and Tang, S.J. (2010) A Comparison of Two Methods for Electrical Conductivity about Plant Leaves. Journal of Guangdong Education Institute, 30, 510-515.

[10] Zhao, S.J., Xu, C.C. and Zou, Q. (1994) Improvements of Method for Measurement of Malondialdehvde in Plant Tissues. Plant Physiology Communications, 30, 207-210.

[11] Wang, Y.R., Liu, H.X., Li, P., Zeng, S.X., Zhen, L.P. and Guo, J.Y. (1986) The Effect of Chilling Stress on Membrane-Lipid Peroxidation of Photosynthetic Apparatus in Rice Seedings in the Dark and Light. Acta Phytophysiologica Sinica, 12, 224-251.

[12] Liu, W.X. and Pan, Y.H. (2007) Sample Preparation Methods Suitable for Wheat Leaf Proteome Analysis. Scientia Agricultura Sinica, 40, 2169-2176.

[13] Zhang, R.Z. and Lu, J.X. (1991) On Morphological and Physiological Characters for Drought Resistance in Rainfed Wheat. Journal of Hebei Agricultural University, 14, 10-14.

[14] Tong, F.P., Fang, W. and Ma, L.Y. (2006) Study on Physiological Response of Protein and Sugar of Slash Pines Halfsib under Water Stress. Chinese Agricultural Science Bulletin, 22, 459-465.

[15] Chen, G., Hu, W.Y. and Xie, P.T. (1991) Solvent for Extracting Malondialdehyde in Plant as an Index of Senescence. Plant Physiology Communications, 109, 2311-2316.

[16] Li, R.Z., Yang, Q.J. and Chen, Y.R. (2004) Study of Determination of Superoxide Dismutase (SOD) Activation and Application. Journal of Qiongzhou University, 11, 321-325.

[17] He, J.Y., Ren, Y.F., Zhu, C. and Jiang, D.A. (2008) Effects of Cadmium Stress on Seed Germination, Seedling Growth, and Amylase Activities in Rice. Chinese Journal of Rice Science, 22, 399-404.

[18] Ding, J.P., Liu, D.M., Zhou, R.Y., Li, F.G. and Li, C.W. (2009) Comparison of Two Purification Methods of Cotton Leaf Total Protein. Journal of Henan Normal University (Natural Science), 37, 103-107. 